\title{
Mejora de la Enseñanza y el Aprendizaje a través de la Evaluación de Competencias por medio de la Herramienta Cycloid
}

\author{
Fernando Julián, Francisco X. Espinach, Manel Alcalà, Andrea Bikfalvi \\ Universitat de Girona, Escola Politècnica Superior, Departamento de Organización, Gestión empresarial y \\ Diseño de Producto.c/ Mำ Aurèlia Capmany 61, 17071 Girona-España. \\ (e-mail: fernando.julian@udg.edu; francisco.espinach@udg.edu; manuel.alcala@udg.edu; \\ andrea.bikfalvi@udg.edu)
}

Recibido May. 23, 2014; Aceptado Ago. 01, 2014; Versión final recibida Sep. 29, 2014

\begin{abstract}
Resumen
El estudio presenta un procedimiento de evaluación y desarrollo de competencias a partir de la utilización de una herramienta basada en las tecnologías de la información y la comunicación, denominada Cycloid. El estudio propone una posible acción para llenar el vacío existente entre la enseñanza universitaria, el aprendizaje y el mercado laboral, observando qué competencias son las más problemáticas para los estudiantes de cara al mercado de trabajo. En el estudio han participado estudiantes de dos universidades, una chilena y otra española. Los resultados muestran que los estudiantes detectan debilidades en la tolerancia al estrés y la competencia lingüística. Sus principales puntos fuertes son la preocupación por el resto del grupo de trabajo y la colaboración mutua. Estos resultados se discuten considerando las evaluaciones anteriores, las acciones e iniciativas correctivas adoptadas y las actividades futuras previstas. Se concluye que los resultados tienen implicancias considerables para los estudiantes, el personal docente y para la gestión universitaria.
\end{abstract}

Palabras clave: cycloid, evaluación de competencias, enseñanza-aprendizaje, TIC's

\section{Improving Teaching and Learning through Assessment Skills using Cyclod}

\begin{abstract}
A procedure for the evaluation and development of competences using a tool based on information and communication technology tool called Cycloid is presented. The study proposes a feasible way for reducing the gap between higher education, learning and the work market, observing which competences were the most problematic for the students that face the work market. Student from two universities, one from Chile and another from Spain, participated in the study. Results show that students show weak stress tolerance and lack language proficiency. Their main strengths are their concern for their teamwork and mutual collaboration. These results are discussed considering the previous evaluations, the corrective and initiative actions adopted and the future proposals of activities. It is concluded that these results have a series of implications mainly for students, teaching staff and university management.
\end{abstract}

Keywords: cycloid, competence evaluation, teaching-learning, ICT's 


\section{INTRODUCCIÓN}

En el presente contexto educativo, aparece la necesidad de acortar la distancia entre la preparación de los estudiantes y las necesidades del mercado laboral. Por lo tanto, las competencias se están convirtiendo en una prioridad para las universidades, ya que este mercado laboral se está centrando en las competencias del personal. Como consecuencia, se han estado integrando en los currículos de aprendizaje (Marín et al., 2013) (Avagliano y Vega, 2013). Ahora, el estudiante debe acreditar el desarrollo de competencias que son potencialmente útiles en las actividades profesionales y a la sociedad en general (Coll y Zegwaard, 2006 y 2011). Se pretende enlazar la formación con las necesidades del mercado laboral (Chang, 2009). El objetivo de este estudio es explorar la relación entre la enseñanza y el aprendizaje, por una parte, y la evaluación de las competencias y su desarrollo, por el otro.

Han participado estudiantes de dos universidades, una chilena y otra española. Por una parte estudiantes del postgrado de Diseño Industrial realizado conjuntamente entre la Universidad de Talca (Chile) y la Universidad de Girona (España), comparándolo con estudiantes de Ingeniería y Diseño de la propia Universidad de Girona, en diferentes cursos académicos. Para completar este objetivo, el estudio pretende responder a tres preguntas principales de investigación: 1) ¿Cuáles son las fortalezas y debilidades de las competencias percibidas en los estudiantes? Esta pregunta da la respuesta a la identificación de posibles áreas de mejora o cambio intencional; 2) ¿Cuáles fueron, respecto a las competencias, los puntos fuertes percibidos de los estudiantes en comparación con los cursos anteriores? Esta pregunta da la respuesta a la identificación de los posibles cambios en términos de competencias. Genera, además, un proceso de análisis de los docentes con el fin de identificar las posibles explicaciones para los cambios en las prioridades de las competencias. ¿Qué grado de similitud se da entre estudiantes de ambas universidades?; y 3) ¿Cuáles son las propuestas de los estudiantes para desarrollar sus debilidades percibidas mediante acciones concretas?

En este trabajo se prueban empíricamente los niveles actuales y los deseados de competencias de los estudiantes de la Universidad de Talca (UT, Chile) que cursan el postgrado en Diseño Industrial de la Universidad de Girona (UdG, España) y la UT, y se comparan con los resultados obtenidos por los alumnos de Ingeniería y Diseño de la misma UdG. La misma metodología se ha utilizado en publicaciones anteriores (Bikfalvi et al., 2007) (Makatsoris, 2009) (Miravitlles et al., 2012), presentando resultados respecto a otros grupos de estudiantes y otros contextos (Bikfalvi et al. 2013). Los datos disponibles sobre las competencias provienen de la auto-evaluación de un grupo de estudiantes seleccionados mediante una herramienta basada en las tecnologías de la información y la comunicación (TIC) llamada Cycloid, cuyo origen se encuentra en la Tampere University of Technology (Finlandia) en el marco general Evolute descrito detalladamente en Kantola et al., $(2005,2011)$. El modelo competencial Cycloid está pensado para puestos de trabajo de gestor de proyectos, definiendo y caracterizando la persona como responsable en la gestión de trabajos complejos, desde la planificación hasta la ejecución y evaluación, con capacidad de interactuar en colaboración con otras personas.

El estudio se centra en la autoevaluación de competencias y la comparación con el nivel que el estudiante considera que desea tener. La aplicación Cycloid, desarrollada y descrita en detalle en (Liikamaa, 2006), trabaja con 30 competencias transversales que tienen una importante relación con las escogidas por la UdG. Cycloid, en entornos académicos, evalúa las competencias en gestión de proyectos, entendiendo como proyecto la realización de los estudios universitarios de los alumnos. La herramienta las estructura en seis grupos principales: conocimiento de uno mismo, autocontrol, capacidad cognitiva, automotivación, empatía y habilidades sociales. Estos 6 grupos se engloban a su vez en otros dos: las competencias personales y las competencias sociales (Tabla 1).

Tabla 1: Modelo de competencias Cycloid (adaptado de Liikamaa (2006).

\begin{tabular}{|l|l|}
\hline \multicolumn{2}{|c|}{ Competencias Personales } \\
\hline Autoconocimiento & Consciencia emocional, confianza en uno mismo, auto evaluación. \\
\hline Autocontrol & $\begin{array}{l}\text { Honradez, mantenimiento orden, innovación, flexibilidad, responsabilidad, búsqueda } \\
\text { información, tolerancia estrés, eficiencia productiva, calidad en decisiones. }\end{array}$ \\
\hline Capacidad Cognitiva & Pensamiento analítico, pensamiento conceptual, competencia lingüística. \\
\hline Automotivación & $\begin{array}{l}\text { Orientación a los objetivos, iniciativa, optimismo, compromiso, comprensión hacia los } \\
\text { otros. }\end{array}$ \\
\hline \multicolumn{2}{|c|}{ Competencias Sociales } \\
\hline Empatia & Desarrollo de los otros, aprovechar la diversidad, conocimiento propia organización. \\
\hline Habilidades Sociales & $\begin{array}{l}\text { Comunicación, gestión de conflictos, dirección, liderazgo, construcción de relaciones, } \\
\text { colaboración, capacidad de grupo. }\end{array}$ \\
\hline
\end{tabular}


El mapa de competencias global se complementa con una hoja de post- evaluación, en donde se recopila la información relativa a las propuestas de los estudiantes en términos de acciones concretas, para mejorar las competencias prioritarias. De acuerdo con la Teoría del Cambio Intencional (Boyatzis y Akrivou, 2006), la comparación entre el yo real (competencia actual) y el yo ideal (objetivo) podría aumentar la conciencia del individuo y actuar como punto de partida para el proceso de cambio intencional y de auto-desarrollo.

\section{METODOLOGÍA}

El presente estudio se centró principalmente en los cursos 2009-10 y 2013-14, realizados en la UdG (España). Los estudiantes estaban matriculados por una parte en el postgrado en Diseño Industrial realizado conjuntamente entre la UT (Chile) y la UdG (España), y por otra parte estudiantes de Ingeniería y Diseño de la propia UdG. Se les pidió que se imaginaran a sí mismos como administradores o gestores de proyectos y evaluar de esta manera la gestión de su curso académico como un proyecto, en este caso de enseñanza-aprendizaje. El estudio se ha realizado en tres etapas claramente diferenciadas: pre-evaluación, evaluación y acciones.

\section{Pre-evaluación}

Consistente en informar a los alumnos sobre el contenido y el funcionamiento de la herramienta. Aproximadamente una semana antes los participantes fueron informados sobre el uso de la aplicación en relación con las competencias. Se les envió una guía donde se expone la aplicación y su utilidad, proporcionando explicaciones sobre las competencias sociales y personales. Se realizó el proceso actuando los estudiantes como directores-gestores del proyecto de su carrera profesional, por medio de preguntas relacionadas con el trabajo diario de los estudiantes.

\section{Evaluación}

Los estudiantes deben valorar 120 afirmaciones relativas a cuestiones cotidianas. Estas afirmaciones engloban los seis grupos de competencias. La aplicación solicita la auto-evaluación del nivel de competencias actual y el nivel de competencias objetivo deseado por el usuario, identificando la posible tensión creativa, es decir, la diferencia entre el estado actual y el deseado. Las respuestas se dan en una escala difusa guiada por etiquetas lingüísticas estándar (por ejemplo: siempre, a menudo, rara vez, nunca) (Kantola et al., 2011). Este modelo identifica un trabajo genérico en gestión de proyectos y analiza la capacidad que tiene una persona para planificar, organizar, dirigir y evaluar trabajos complejos o proyectos que requieren una interacción sólida y frecuente con un equipo humano, con el fin de planificar todo el proyecto (Müller y Turner, 2010).

Inmediatamente después de completar la encuesta, de forma adicional se les entregó una descripción de las competencias para su comprensión y una hoja para que anotaran propuestas para el plan de desarrollo individual de las tres competencias que desean mejorar. Esta forma invita a los estudiantes a proponer acciones concretas para el desarrollo de competencias. Sobre la base de sus resultados individuales se les pide cumplimentar tres acciones o actividades concretas que piensan que les serían útiles para mejorar sus debilidades percibidas. La evaluación se ha llevado a cabo en una sesión de clase regular en un aula informatizada con acceso a Internet. El proceso se ha seguido por parte del profesorado y el administrador Cycloid para cualquier asistencia técnica relacionado con la utilización de la plataforma.

La evaluación genera el informe competencial individual que contiene las 30 competencias del gestor de proyectos. Posteriormente el usuario ordena su informe siguiendo diversos criterios, como por ejemplo el de establecer las diferencias en cuanto a tensión creativa. La utilización de soluciones de lógica borrosa (fuzzy logic) permite que los alumnos puedan valorar libremente su nivel de competencias actuales y sus aspiraciones, dentro de una escala semántica continua. No se presenta el algoritmo de cómo se calcula el valor numérico de la tensión creativa, aunque es el concepto según el cual el informe competencial proporciona información de mayor utilidad. A nivel conceptual, representa la diferencia entre el "estado objetivo" y el "estado actual" de cada una de las competencias. Su contenido se alimenta de aproximadamente 4 afirmaciones por cada una de las competencias. El estudio se puede replicar utilizando la misma plataforma (véase los estudios citados previamente que utilizan la misma herramienta).

\section{Acciones}

En el caso de los estudiantes de postgrado de la UT, el curso 2009-10 la totalidad de los alumnos (23), realizaron el estudio. El curso 2013-14 igualmente todos los alumnos matriculados, en este caso 16, completaron el estudio, generando alrededor de 69 acciones para el primer caso y 48 para el segundo. Respecto a los estudiantes de la UdG, 56 alumnos de último año completaron la evaluación de las 
competencias el curso 2009-10 de los cuales 32 corresponden a Ingeniería y diseño, siendo 40 el número de matriculados y 24 corresponden a Ingeniería informática, siendo en este caso 46 el número de matriculados. En relación a los estudiantes de Ingeniería agrícola, el curso 2013-14 de los 54 estudiantes matriculados, completaron la evaluación 27, generando alrededor de 80 acciones.

\section{RESULTADOS Y DISCUSIÓN}

La figura 1 presenta los resultados detallados del curso 13/14 respecto a los alumnos del postgrado en diseño (UT). Las 30 competencias están dispuestas en un orden decreciente de tensión creativa, lo que representa la voluntad individual hacia el cambio. Una lectura interpretativa de los resultados muestra que:

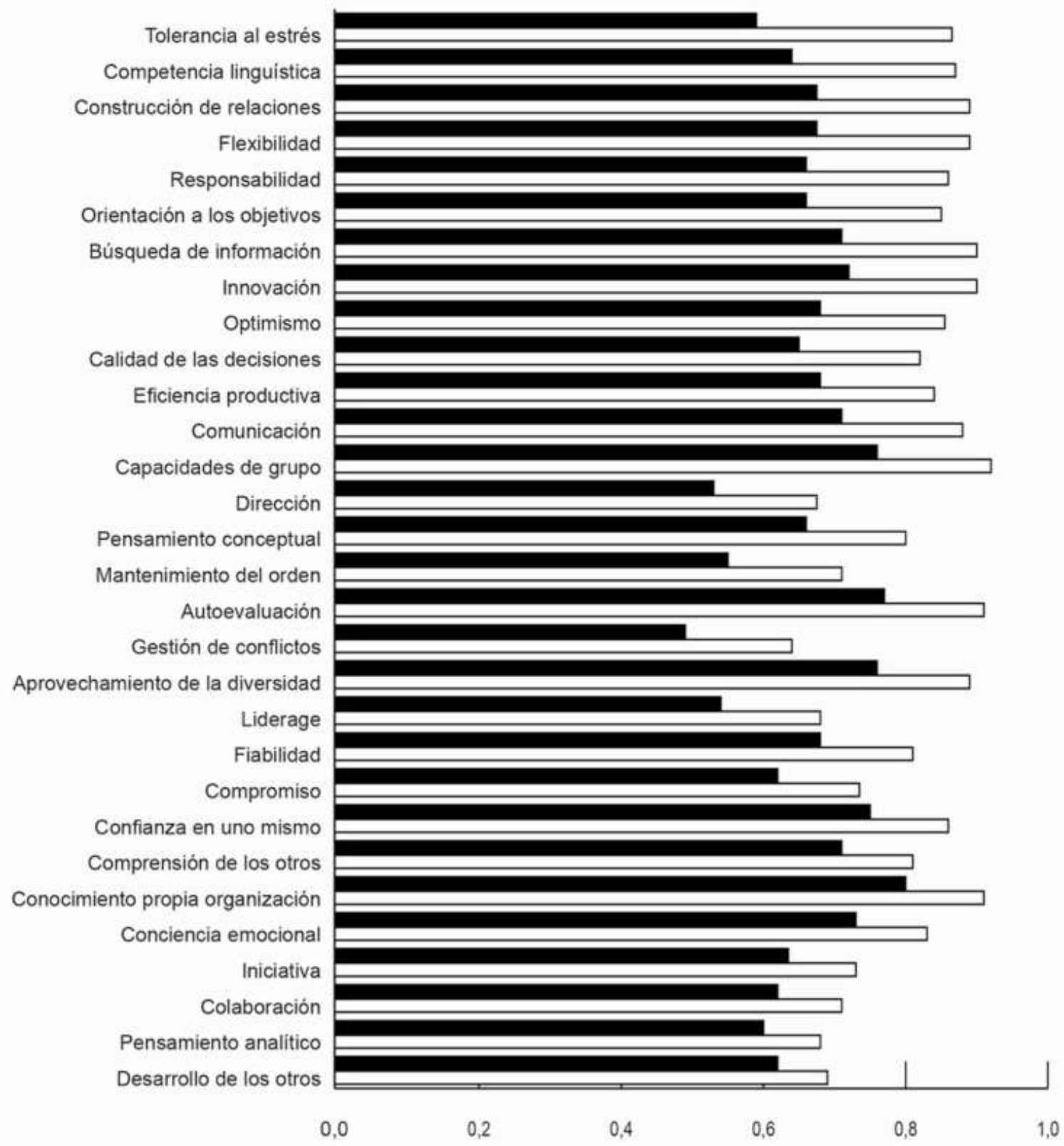

Fig. 1: Resultados curso 13/14 correspondientes a los alumnos del postgrado en diseño (UT). Los colores significan: $\square$ ojetivo y $\square$ actual

1) Las debilidades percibidas de los estudiantes, o aquellas competencias para las que perciben una brecha de mejora mayor (situadas en la parte superior de la tabla), son: Tolerancia al estrés (capacidad de manejar los asuntos y situaciones desfavorables, agotador y estresante y las emociones fuertes), la competencia lingüística (habilidad y coraje en el uso de lenguas extranjeras), la construcción de relaciones (establecimiento, mantenimiento y desarrollo de relaciones beneficiosas y redes no oficiales) y la flexibilidad (actitud flexible respecto a los cambios y la diversidad). 
2) Respecto a las fortalezas percibidas por los estudiantes, o aquellas competencias para las que perciben una brecha de mejora más pequeña (situadas en la parte inferior del gráfico), son: desarrollo de los otros (Identificación de las necesidades de desarrollo de los otros y promoción de sus capacidades), pensamiento analítico (división de los problemas en partes y organización sistemática de las partes a partir de bases racionales), y colaboración (Trabajo en equipo para conseguir objetivos comunes).

En el curso 2009-10 se realizó el mismo estudio (Espinach et al., 2010). En aquel caso participaron 23 estudiantes y el resultado mostró respecto a las debilidades percibidas los siguientes datos: Tolerancia al estrés, la competencia lingüística, responsabilidad (conciencia y compromiso de las acciones propias) y construcción de relaciones. Respecto a las fortalezas percibidas por los estudiantes: Iniciativa (percepción, aprovechamiento y habilidad para crear nuevas oportunidades), colaboración, desarrollo de los demás y pensamiento analítico. Como puede observarse, el resultado en ambos casos es prácticamente similar.

En el mismo curso 2009-10 se realizó el mismo estudio comparando estudiantes de dos universidades españolas, la UdG y la Universidad de Zaragoza (UZ), ubicadas en diferentes territorios o regiones. En este caso los estudiantes cursaban el grado en Ingeniería en Diseño. Participaron por parte de la UdG 30 estudiantes y por parte de la UZ, 34. El estudio puede verse en Julián et al., (2011) arrojando resultados similares. Otros estudios sobre análisis de competencias en estudiantes de Ingeniería del diseño de otras universidades españolas puede encontrarse en Agudo et al., (2013).

En ese mismo curso académico 2009-10 se realizó igualmente el estudio a alumnos de Ingeniería industrial y de ingeniería informática de la UdG que puede consultarse en Verdaguer et al., (2010). En el primer caso participaron 32 estudiantes y en el segundo, 24. En el caso de los estudiantes de Ingeniería industrial, las debilidades percibidas fueron: Tolerancia al estrés, competencia lingüística y calidad de las decisiones. Como puede observarse una situación similar a las anteriores. En el caso específico de los estudiantes de Ingeniería informática las debilidades percibidas son prácticamente las mismas, destacando en segundo lugar, en este caso, la construcción de relaciones.

Los primeros estudios realizados con la herramienta Cycloid, por el grupo de investigación, al cual pertenece el presente trabajo, datan de los cursos 2007-8 y 2008-09, en donde se analizaron la tensión creativa aplicada al análisis de competencias a estudiantes de Ingeniería agrícola y de diseño de la UdG. El estudio puede consultarse en (Alcalà et al., 2010). Los resultados arrojados fueron similares a los comentados anteriormente.

En el curso académico 2013/14 (Figura 2) se realizó el estudio a estudiantes de ingeniería agrícola de la UdG, que al igual que en los estudiantes de postgrado en diseño se les había hecho el mismo estudio el curso 2009/10:

1) Las debilidades percibidas de los estudiantes, o aquellas competencias para las que perciben una brecha de mejora mayor, fueron: Capacidades del grupo (creación de sinergias, llegar a metas comunes), la iniciativa, confianza en sí mismo (la creencia en la propia capacidad de uno), la honradez (la honestidad, la ética profesional), calidad de las decisiones (decisiones basadas en principios y valores) y de comunicación.

2 ) Respecto a las fortalezas o aquellas competencias para las que perciben una brecha de mejora más pequeña o incluso negativa, fueron: la tolerancia al estrés, el optimismo (alcanzando metas a pesar de los obstáculos y reveses), el aprovechamiento de la diversidad (la consecución de objetivos junto con una diversidad de personas), el pensamiento conceptual (reconocimiento, la aplicación y la definición de los conceptos) y la eficiencia de la producción (la realización de tareas de forma rápida y eficaz).

Estos resultados dan respuesta a Q1 planteada como "¿Cuáles son las fortalezas y debilidades de las competencias percibidas de los estudiantes para el curso 2013/14?". Los resultados previos, en evaluaciones realizadas en años académicos anteriores, muestran un cambio significativo en términos de competencias, tales como la tolerancia al estrés y dominio de la lengua, como piezas de ejemplo. Cambio producido en los estudiantes de la UdG respecto a los de la UT. Tradicionalmente aparecen como principales debilidades, en cambio para estos estudiantes aparecen como puntos fuertes. Puede observarse por tanto, un cambio importante. Tanto los alumnos de ingeniería de la UdG como los de postgrado (UTUdG) obtenían tradicionalmente los mismos resultados, al igual que otros cursos de ingeniería de la UdG. Se produce un cambio revelador que puede ser debido a una serie de acciones realizadas por parte de algunos docentes.

A modo de resumen, la tabla 2 visualiza las cinco fortalezas y debilidades de ambos grupos de estudiantes. Los resultados muestran al menos los siguientes aspectos: 1) diferentes grupos de estudiantes (aunque sean de la misma carrera o carreras similares) tienen perfiles competenciales diferentes, 2) una competencia, aunque sea la misma, se percibe como debilidad por unos y como fortaleza por otros. 


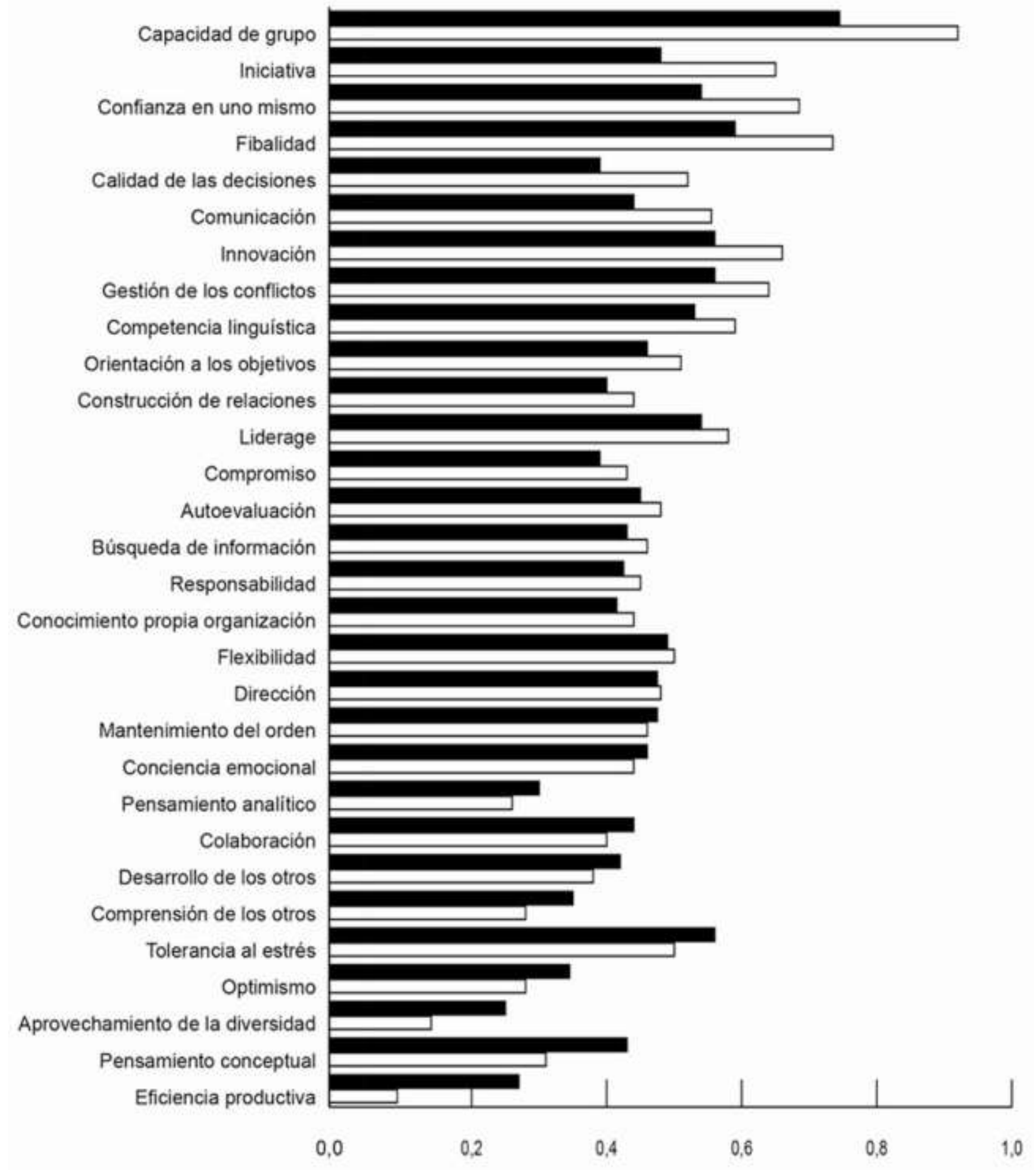

Fig. 2: Resultados curso 13/14 respecto a los alumnos de Ingeniería agrícola (UdG).

Los colores significan: $\square$ ojetivo y $\mathbf{\square}$ actual

Una vez iniciado el proceso de evaluación de competencias y obtenidos los primeros resultados, el curso 2009/2010, el equipo de profesores reflexionó sobre cómo podría ayudar al alumnado a mejorar las competencias percibidas como debilidades en la mayoría de asignaturas, en el marco de las cuales se introdujo la evaluación de competencias. A partir de los informes competenciales grupales, el equipo docente diseñó y aplicó gradualmente acciones esperadas de mejora. La Tabla 3 muestra las principales novedades introducidas por los educadores desde 2010 enfocadas a la competencia de tolerancia al estrés, la cual históricamente y en todos los contextos ha sido percibida como de mayor debilidad. 
Tabla 2: Fortalezas y debilidades más destacadas del curso 13/14

\begin{tabular}{|c|c|}
\hline $\begin{array}{c}\text { Postgrado en Diseño } \\
\text { (UdG-UT) }\end{array}$ & $\begin{array}{c}\text { Ingeniería Agrícola } \\
\text { (UdG) }\end{array}$ \\
\hline Tolerancia al estrés & Fortalezas \\
\hline Competencia lingüística & Conciencia emocional \\
\hline Construcción de relaciones & Iniciativa \\
\hline Flexibilidad & Colaboración \\
\hline Responsabilidad & Pensamiento analítico \\
\hline & Desarrollo de los otros \\
\hline Capacidad de grupo & Tolerancia al estrés \\
\hline Iniciativa & Optimismo \\
\hline Confianza en uno mismo & Aprovechamiento de la diversidad \\
\hline Fiabilidad & Pensamiento conceptual \\
\hline Calidad de las decisiones & Eficiencia productiva \\
\hline
\end{tabular}

Tabla 3: Principales acciones introducidas por los educadores.

\begin{tabular}{|c|l|}
\hline Acción & \multicolumn{1}{c|}{ Descripción } \\
\hline $\begin{array}{c}\text { Asunto a } \\
\text { planificar }\end{array}$ & $\begin{array}{l}\text { Se ha desarrollado y aplicado una estructura. Los contenidos se han organizado en grupos } \\
\text { temáticos. Todos los criterios y la carga de trabajo asumida respecto al tema han sido } \\
\text { compartidos y se ha presentado a los estudiantes el primer día de clase. Se ha publicado } \\
\text { en la Intranet. }\end{array}$ \\
\hline $\begin{array}{c}\text { Planificación } \\
\text { personal }\end{array}$ & $\begin{array}{l}\text { Se ha compartido con cada estudiante un documento con respecto a las fechas de entrega } \\
\text { y el contenido de todas las tareas y asignaciones en el tema. }\end{array}$ \\
\hline $\begin{array}{c}\text { Entrega de } \\
\text { trabajos }\end{array}$ & $\begin{array}{l}\text { Entrega de trabajos en asignaturas como Expresión Gráfica a la Ingeniería. Se han } \\
\text { dividido las entregas de los trabajos: parcialmente en el aula y en parte fuera del aula. } \\
\text { Todos los detalles y los plazos están definidos y compartidos con los estudiantes. }\end{array}$ \\
\hline Proyecto & $\begin{array}{l}\text { Proyecto basado en la metodología PBL. El profesor requiere equipos de estudiantes (2-3) } \\
\text { para desarrollar un proyecto orientado a un producto. El objetivo principal es demostrar y } \\
\text { aplicar en la práctica el contenido explicado. Se solicita un informe para ser evaluado. } \\
\text { Representa el 15 \% de la nota final. }\end{array}$ \\
\hline
\end{tabular}

Aún no hay posibilidades de demostrar una clara relación causa-efecto por falta de disponibilidad de datos cuantitativos, pero los autores creen firmemente que las medidas adoptadas han contribuido positivamente al cambio descrito. Por lo tanto, la respuesta a Q2 "¿Cuáles fueron, respecto a las competencias, los puntos fuertes percibidos de los estudiantes en comparación con los cursos anteriores?" La respuesta es que respecto a los estudiantes de la UdG son diferentes y aún se podría decir más, contrapuestas a años anteriores. Competencias que han estado en la cima de las consideraciones de los estudiantes con respecto a sus debilidades están apareciendo como sus principales puntos fuertes. Esto significa además, que las prioridades de los estudiantes cambian con los años y los educadores deben adaptarse con acciones concretas que aborden estos desafíos. Cada vez es más compleja una planificación, y es necesaria la mejora de contenidos y sistemas de evaluación novedosos con el fin de subsanar las deficiencias de desarrollo.

La respuesta a "¿Qué grado de similitud se da entre estudiantes de ambas universidades? La respuesta indica que era la misma hasta que se realizaron acciones sobre uno de los grupos. Complementando la iniciativa de los educadores, se pidió a los estudiantes que propusieran tres actividades concretas asociadas a 3 competencias que consideraran una prioridad. Cada estudiante tenía que proponer por escrito un total de nueve acciones que estarían dispuestos a aplicar con el fin de mejorar. Puesto que no se limitaban a las restricciones, las entradas resultantes son muy variadas. La tabla 4 contiene los resultados más destacados de las 5 competencias que consideran una prioridad y para los que el grupo de Ingeniería proporcionó la mayoría de las respuestas. 
Tabla 4: Acciones de los estudiantes para el desarrollo de competencias

\begin{tabular}{|c|c|c|}
\hline Competencia & $\begin{array}{l}\text { NNo acciones } \\
\text { /propuestas }\end{array}$ & Acciones de ejemplo \\
\hline Iniciativa & $28 / 10$ & $\begin{array}{l}\text { Proponer nuevas ideas; el trabajo sobre los proyectos; crear nuevos objetivos; } \\
\text { proponer casos; crear nuevos hábitos; no copiar otros trabajos; pensar y } \\
\text { analizar situaciones } 15 \text { minutos / día; participar más en los debates; hacer más } \\
\text { en clase; trabajar en proyectos innovadores; hacer proyectos individuales. }\end{array}$ \\
\hline $\begin{array}{c}\text { Confianza en } \\
\text { uno mismo }\end{array}$ & $26 / 9$ & $\begin{array}{l}\text { Expresar y compartir los sentimientos; despertar en la mañana con una alta } \\
\text { motivación; ir al psicólogo; practicar la auto-motivación; leer todas las mañanas } \\
\text { una frase de motivación; no pienses "yo no puedo hacer"; tratar de realizar } \\
\text { tareas difíciles de demostrar a mí mismo "yo puedo". }\end{array}$ \\
\hline $\begin{array}{c}\text { Capacidades } \\
\text { de grupo }\end{array}$ & $25 / 10$ & $\begin{array}{l}\text { Incrementar el trabajo en grupo; participar en los debates; incrementar las } \\
\text { relaciones humanas y la interacción; participar y discutir colectivamente sobre } \\
\text { un tema; hacer grupos de clase más pequeños; realizar evaluaciones de los } \\
\text { grupos más destacados; actividades de ocio con compañeros de clase; estudiar } \\
\text { con compañeros de clase. }\end{array}$ \\
\hline $\begin{array}{c}\text { Competencia } \\
\text { lingüística }\end{array}$ & $22 / 8$ & $\begin{array}{l}\text { Asistir a clases de academia privada; practicar idioma extranjero cada día; con } \\
\text { frecuencia hacer ejercicios y hablar; ver la televisión en el idioma original; leer } \\
\text { más; hablar con extranjeros; enseñar a otros idiomas; asistir a actividades en las } \\
\text { que sólo se puede practicar idiomas extranjeros. }\end{array}$ \\
\hline Comunicación & $20 / 7$ & $\begin{array}{l}\text { Hacer más exposiciones orales; ser más abiertos y hablar más con la gente; } \\
\text { conocer personas nuevas; asistir a fiestas; asistir a terapias de grupo; ser claro y } \\
\text { directo; expresar mi propia opinión y escuchar a los demás; conocer y trabajar } \\
\text { junto con compañeros de clase; actividades conjuntas; la interacción social. }\end{array}$ \\
\hline
\end{tabular}

Este ejercicio es interesante en términos de iniciativa y eficacia. Incluso los educadores y docentes son capaces de facilitar las acciones. En ellas se detecta la propuesta por parte del estudiante de lo que podría ser más apropiado, ya que es su voluntad y preferencia hacia las acciones más representativas de sus intereses. Mientras tanto, también se enfrentan a procesos de toma de decisiones y cultivo de la iniciativa propia. La iniciativa, se encuentra entre las competencias más deseadas para desarrollar o mejorar.

Es interesante observar que la mayoría de las propuestas de los estudiantes son coherentes y en línea con las competencias. La mayoría de las veces, están orientadas hacia el estudio y no tanto hacia el ocio. Otra observación notable es que las competencias seleccionadas están relacionadas con acciones que no están totalmente en línea con los resultados del grupo proporcionadas por la herramienta de evaluación. Esto se puede observar mediante la comparación de los resultados en la Figura 2 y los que muestra la Tabla 4. Adicionalmente a los puntos débiles que aparecen en ambos casos, los estudiantes apuntan al dominio del idioma como aspecto fuerte en la Tabla 4. Esto significa además que la disposición de los estudiantes hacia la mejora y la importancia de esta competencia son signos hacia el cambio intencional. En general, se observa también una actitud muy positiva durante la evaluación y el ejercicio de las acciones propuestas. Los estudiantes se sienten como parte de este proceso de transformación compleja y se les informa que su opinión importa, siendo éste un canal de gran alcance para facilitarlo. Se han propuesto y completado todas las formas coherentes de corrección de las acciones. Esto demuestra cierta madurez y seriedad. Dado que el ejercicio es anónimo, con el fin de aumentar la sinceridad de la participación, es imposible relacionarlo con el informe de la competencia generada por la plataforma Cycloid. Por lo tanto, esta sección proporciona la respuesta a Q3 planteada como "¿cuáles son las propuestas de los estudiantes para desarrollar sus debilidades percibidas mediante acciones concretas? " Tanto en términos de acciones concretas como algunas de sus características, éstas fueron: la coherencia, lo concreto, orientado al aprendizaje simple, aplicable... entre otras.

Tradicionalmente la enseñanza y el aprendizaje en la educación superior se han centrado en la evaluación de conocimientos. En el escenario actual, no sólo el conocimiento, sino también la evaluación de competencias junto con su desarrollo correcto, son un reto tanto para las instituciones de educación superior (IES) como para los educadores. Las tecnologías TIC sirven de apoyo técnico y actúan como un poderoso facilitador para generar todo tipo de ambientes "electrónicamente conectados" para que estudiantes y educadores se adapten continuamente. En este trabajo se presentan los resultados de una autoevaluación de competencias y de las iniciativas propuestas por los propios estudiantes con el fin de 
mejorar las que están peor posicionadas, obtenidas en el informe global de su autoevaluación. Las implicaciones de este estudio son considerables para todas las partes involucradas en el proceso de enseñanza-aprendizaje y se detallan a continuación.

Estudiantes: Se convierten en parte activa, no son sólo un objetivo del proceso de aprendizaje y transformación de la asignatura. En primer lugar, su curiosidad hacia los resultados inmediatos les conduce hacia la evaluación. Reciben un informe de sus competencias, un nuevo enfoque en donde se refleja su propia visión en términos de las competencias actuales y futuras. En segundo lugar, se dan cuenta de la importancia del desarrollo de las competencias y que (como individuos), junto con el profesorado, son elementos activos en sus logros. En tercer lugar, se familiarizan con una herramienta muy similar a una prueba de evaluación de competencias comunes en las empresas, ya sea en el acceso como a la hora de determinar sus necesidades de formación. Por último, el auto-conocimiento como una condición previa a la auto-motivación, es un ingrediente importante para el entorno de enseñanza-aprendizaje orientado al éxito.

Educadores: Pueden desarrollar una enseñanza más centrada en el estudiante con el fin de contribuir a un desempeño eficaz, útil y diferenciado, tanto en los centros educativos como en los entornos de futuros trabajos. La integración de la evaluación de competencias y el desarrollo tradicional son un tema complejo y con frecuencia los educadores carecen de conocimientos y experiencia en este campo. Puede cambiarse la realización de pruebas de evaluación como una necesidad. Como sustituto, el uso de la herramienta TIC presentada previamente, ofrece un mapa de competencias colectivas en el que se puede alcanzar y aportar información valiosa y novedosa en lo que son las necesidades del grupo-clase. Proponer acciones de los estudiantes, que permita a los profesores ver qué tipo de actividades están más dispuestos a realizar.

Gestión de la Universidad: Escuchando y siendo conscientes de las necesidades de los estudiantes, la gestión universitaria puede aumentar la satisfacción de sus futuros clientes o alumnos. Este aspecto está en consonancia con un enfoque más orientado a los negocios en los que la satisfacción del usuario de los servicios y la competitividad resultante, son caminos de futuro para las IES pequeñas, de tamaño medio, regionales y periféricas. Otro reto es la gestión correcta y la integración entre la variedad e incremento de herramientas TIC que sirven para diferentes objetivos (software de diseño, software Moodle para los contenidos y evaluaciones, software ACME para el aprendizaje continuo y la evaluación).

Los procesos de transformación a menudo tardan un tiempo y son el resultado de un cúmulo de factores. Complementado con la realidad de que la mayoría de los estudiantes cambian cada curso (año académico se hace difícil determinar hasta qué grado los cambios en las prioridades de desarrollo de competencias son el resultado directo de los cambios en la asignatura.

\section{CONCLUSIONES}

Tradicionalmente los estudiantes detectan debilidades principalmente en la tolerancia al estrés y la competencia lingüística. Al contrario, sus principales puntos fuertes son el desarrollo de los demás y la colaboración. La introducción de acciones concretas por parte de los docentes probablemente puede contribuir a modificar las competencias más débiles. Los resultados detallados tienen implicaciones importantes para: estudiantes, personal docente y gestión universitaria. La principal limitación del presente estudio consiste en la complejidad del tema abordado en términos de transformación de las asignaturas asociadas a las necesidades del grupo y el hecho de basarlas en sus competencias.

\section{REFERENCIAS}

Agudo, J.E.; R. Hernández-Linares; M. Rico y H. Sánchez. Competencias Transversales: Percepción de su desarrollo en el Grado en Ingeniería en Diseño Industrial y Desarrollo de Productos, Formación Universitaria, 6 (5), 39-50 (2013).

Alcalà, M.; F. Julián; F.X. Espinach y N. Verdaguer. Tensión creativa aplicada al análisis de competencias a alumnos de ingeniería, Formación Universitaria, 3 (3), 23-32 (2010).

Avagliano, A.R. y S.A. Vega. Mejora del Proceso de Enseñanza y Aprendizaje en la Carrera de Ingeniería de Ejecución Mecánica. Diseño Micro-curricular Basado en Resultados de Aprendizaje. Formación Universitaria, 6 (4), 3-12 (2013).

Bikfalvi, A.; J. Llach; J. Kantola; P. Marques y N. Mancebo. Complementing education with competence development: An ICT-based application, Int. Journal of Management in Education, 1, 231-250 (2007).

Bikfalvi, A.; A. Rafart y N. Mancebo. The Assessment of Competence in University Adult Education: A Dynamic Approach, International Journal of Management, Knowledge and Learning, 1(2), 3-22 (2013) 
Boyatzis, R. y K. Akrivou. The ideal self as the driver of intentional change, Journal of Management Development, 25, 624-642 (2006).

Chang, Y.; T. Eklund; J. Kantola y H. Vanharanta. International Creative Tension, Study of University students in South Korea and Finland, Human Factors and Ergonomics in Manufacturing, 19 (6), 528-543 (2009).

Coll, R.K. y K. Zegwaard. Perceptions of desirable graduate competencies for science and technology new graduates, Research in science and Technological Education, 24 (1), 29-58 (2006).

Coll, R.K. y K. Zegwaard. Exploring some current issues for Cooperative Education, Journal of Cooperative Education \& Internships, 45 (2), 8-15 (2011).

Espinach, F.X.; F. Julián; M. Alcalà y N. Verdaguer. Our experience with an ICT Application to analysis competences, Proceedings of ICERI2010 Conference, 6761-6766, Madrid, España, 15-17 Nov. (2010).

Julián, F.; F.X. Espinach; A. Serrano; I. Lidón y R. Rebollar. Estudio de la tensión creativa en estudiantes de Ingeniería de dos universidades españolas, Interciencia, 36 (7), 524-530 (2011).

Kantola, J.; H. Vanharanta y W. Karwowski. The evolute system: A co-evolutionary human resource development methodology, Int. Encyclopedia of Human Factors and Ergonomics, 3, 2894-2900 (2005).

Kantola, J.; W. Karwowski y H. Vanharanta. Managing managerial mosaic: the Evolute methodology, Enterprise Information Systems and Implementing IT Infrastructures. Challenges and Issues. IGI Global (2011).

Liikamaa, K. Tacit knowledge and project managers' competences, Doctoral thesis, Tampere University of Technology at Pori, Finland (2006).

Makatsoris, C. An information and communication technologies-based framework for enhancing project management education through competence assessment and development, Human Factors and Ergonomics in Manufacturing \& Service Industries, 19(6), 544-567 (2009).

Marín, R.; I. Guzmán; A. Márquez y M. Peña. La Evaluación de Competencias Docentes en el Modelo DECA: Anclajes Teóricos Marín, Formación Universitaria, 6 (6), 41-54 (2013).

Miravitlles-Matamoros, P.; A. Nunez-Carballosa; P. L. Guitart-Tarres; F. Achcaoucaou-lallouchen; M. Bernardo, y E. Hormiga. Implementation of an ICT-based tool for assessing students competences in business administration subjects. Proceedings of INTED2012 Conference, 5276-5283, Valencia, España, 57 Marzo (2012).

Müller, R. y R. Turner. Leadership competency of successful project managers, International Journal of Project Mangement, 28, 437- 448 (2010).

Verdaguer, J.; M. Alcalà; F. Julián y F.X. Espinach. An analysis of competences among university studies and students perception. Proceedings of EDULEARN10 Conference, 4052-4060, Barcelona, España, 5-7 Julio (2010). 